\title{
NEW BOUNDS FOR DISTANCE ENERGY OF A GRAPH
}

\author{
G. SRIDHARA ${ }^{1}$, M. R. RAJESH KANNA ${ }^{2}$ AND \\ H L PARASHIVAMURTHY ${ }^{3}$ \\ ${ }^{1}$ Post Graduate Department of Mathematics, \\ Maharani's Science College for Women, \\ J. L. B. Road, Mysore - 570 005, India. \\ e-mail:srsrig@gmail.com \\ ${ }^{2}$ Department of Mathematics, \\ Sri D Devaraj Urs Government First Grade College, \\ Hunsur - 571105, India. \\ e-mail:mr.rajeshkanna@gmail.com \\ ${ }^{3}$ Research Scholar, \\ Research and Development Centre, \\ Bharathiar University, Coimbatore - 641 046, India. \\ and \\ BGS Institute of Technology, B.G Nagar, Bellur- 571448, India. \\ e-mail:hlpmathsbgs@gmail.com
}

\begin{abstract}
For any connected graph $G$, the distance energy, $\mathcal{E}_{D}(G)$ is defined as the sum of the absolute eigenvalues of its distance matrix. Distance energy was introduced by Indulal et al. in the year 2008 [10]. It has significant importance in QSPR analysis of molecular descriptor to study their physico-chemical properties. Our interest in this article is to establish new lower and upper bounds for distance energy.

Key words and Phrases: Distance matrix, Wiener index, Bounds for distance energy of a graph.
\end{abstract}

\section{INTRODUCTION}

In chemistry, Huckle molecular Orbital(HMO) theory is used to calculate $\pi$-electron energy of conjugated hydrocarbon. Later it was proved this quantity is equivalent to $\mathcal{E}(G)=\sum_{i=1}^{n}\left|\lambda_{i}\right|$, where $\lambda_{1} \geq \lambda_{2} \geq \cdots \geq \lambda_{n}$ are eigenvalues of the respective molecular graph and called it as energy of graph. The studies on

2020 Mathematics Subject Classification: 05C50, 05C69.

Received: 07-12-2018, accepted: 23-02-2020. 
graph energy can be seen in papers [5,6]. For detailed survey on applications on graph energy, see papers $[2,3,4,7]$. The bounds for $\mathcal{E}(G)$ can be found in papers $[12,13,14,11]$.

In what follows in this paper, we take the graph $G$ as simple undirected graph $G$ with $n$ vertices and $m$ edges. For any two vertices $v_{i}$ and $v_{j}$, the distance between them is denoted by $d_{i j}$ and is defined as the shortest path from $v_{i}$ to $v_{j}$. Two parameters that are of interest are Wiener index, $W(G)$ and distance matrix $A_{D}(G)$. They are respectively defined by $W(G)=\sum_{i<j} d_{i j}$ and $A(G)=A_{D}(G)=\left[d_{i j}\right]$. For the sake of simplicity Wiener index is written as $W$. Clearly $A_{D}(G)$ is a symmetric matrix, its eigenvalues are root of equation $\phi(G: \mu)=|\mu I-A(G)|=0$. These eigenvalues are called $D$-eigenvalues or $D$-spectrum which are generally ordered in the form $\mu_{1} \geq \mu_{2} \geq \cdots \geq \mu_{n}$. The largest eigenvalue $\mu_{1}$ is called the distance spectral radius of the graph $G$. Given a graph $G$, the distance energy of $G$ is defined by $\mathcal{E}_{D}(G)=\sum_{i=1}^{n}\left|\mu_{i}\right|$.

For a connected graph $G$, Koolen and Moulton upper bound [8] for distance energy in terms of $W, M$ and $n$ is

$$
\mathcal{E}_{D}(G) \leq\left(\frac{2 W}{n}\right)+\sqrt{(n-1)\left(2 M-\left(\frac{2 W}{n}\right)^{2}\right)} \text { for } 2 W \geq n
$$

where $M=\sum_{i<j}^{n} d_{i j}^{2}$. Further results on upper bounds can also seen in the paper [9].

McClelland bounds [8] for distance energy of graph which is true for any connected graph $G$

$$
\sqrt{2 M+n(n-1)|\operatorname{det}(A)|^{\frac{2}{n}}} \leq \mathcal{E}_{D}(G) \leq \sqrt{2 M n} .
$$

For all studies on distance energy refer papers $[1,10,15]$. We use the following two lemmas, which followed from the properties of distance eigenvalues [8].

Lemma 1.1. Let $G$ be a graph with $n \geq 3$ vertices and $m$ edges. Let $\mu_{1} \geq \mu_{2} \geq$ $\cdots \geq \mu_{n}$ be $D$-eigenvalues of $G$ then

$$
\sum_{i=1}^{n} \mu_{i}=0
$$

and

$$
\sum_{i=1}^{n} \mu_{i}^{2}=2 M
$$


Lemma 1.2. If $\mu_{1}(G)$ is distance spectral radius of the graph $G$ then $\mu_{1}(G) \geq \frac{2 W}{n}$. Since $2 W \geq n, \mu_{1} \geq 1$.

Throughout this paper, during proof of the theorems we use notations $M=\sum_{i<j}^{n} d_{i j}^{2}$ and $A_{D}(G)=A$. Note that $M=\sum_{i<j}^{n} d_{i j}^{2} \geq \sum_{i<j}^{n} d_{i j}=W$ and $\sqrt{M}=\sqrt{\sum_{i<j}^{n} d_{i j}^{2}} \leq \sum_{i<j}^{n} d_{i j}=W$.

\section{MAIN RESULTS}

\subsection{Lower bound for spectral distance radius.}

Lemma 2.1. If $A$ is adjacency distance matrix of a graph $G$ with $n$ vertices and $m$ edges then

$$
|\operatorname{det}(A)| \leq(2 M)^{\frac{n}{2}}
$$

Proof. Derivation follows from $|\operatorname{det}(A)|=\left|\mu_{1} \mu_{2} \ldots \mu_{n}\right|=\left|\mu_{1}\right|\left|\mu_{2}\right| \ldots\left|\mu_{n}\right|$. But

$$
|\operatorname{det}(A)| \leq\left|\mu_{1}\right|\left|\mu_{1}\right| \ldots\left|\mu_{1}\right|=\left|\mu_{1}\right|^{n} \leq(\sqrt{2 M})^{n} .
$$

This gives $|\operatorname{det}(A)| \leq(2 M)^{\frac{n}{2}}$.

Lemma 2.2. If $G$ is a connected graph with $n$ vertices and $m$ edges then the largest distance eigenvalue, $\mu_{1}$ of $G$ satisfies

$$
\left|\mu_{1}\right| \geq|\operatorname{det}(A)|^{\frac{1}{n}}
$$

Proof. Using the relation $\mu_{1}+\mu_{2}+\cdots+\mu_{n}=0$ on distance eigenvalues of the graph $G$ gives $\mu_{2}+\cdots+\mu_{n}=-\mu_{1}$. Since $\mu_{1} \geq 1$, the sum $\mu_{2}+\cdots+\mu_{n}$ is negative quantity. Therefore

$$
\mu_{2}+\cdots+\mu_{n} \leq\left|\mu_{2} \mu_{3} \cdots \mu_{n}\right|^{\frac{1}{n-1}}
$$

i.e.

which implies

$$
-\mu_{1} \leq \frac{\left|\mu_{1} \mu_{2} \cdots \mu_{n}\right|^{\frac{1}{n-1}}}{\mu_{1}^{\frac{1}{n-1}}}
$$

$$
-\mu_{1}^{\frac{n}{n-1}} \leq|\operatorname{det}(A)|^{\frac{1}{n-1}}
$$

So,

$$
\left|\mu_{1}\right|^{\frac{2 n}{n-1}} \leq|\operatorname{det}(A)|^{\frac{2}{n-1}}
$$

if $\left|\mu_{1}\right| \leq 1$ and $\left|\mu_{1}\right|^{\frac{2 n}{n-1}} \geq|\operatorname{det}(A)|^{\frac{2}{n-1}}$ if $\left|\mu_{1}\right| \geq 1$. But $\left|\mu_{1}\right| \geq 1$. Hence $\left|\mu_{1}\right| \geq|\operatorname{det}(A)|^{\frac{1}{n}}$. 
Lemma 2.3. If $G$ is a graph with $n$ vertices and $m$ edges then the largest distance eigenvalue, $\mu_{1}$ of $G$ satisfies

$$
\left|\mu_{1}\right| \geq \frac{|\operatorname{det}(A)|^{\frac{1}{n}}}{\sqrt{n}} .
$$

Proof. Arithmetic and geometric mean of $\left|\mu_{1}\right|,\left|\mu_{2}\right|, \cdots,\left|\mu_{n}\right|$ are respectively are

$$
\frac{\left|\mu_{1}\right|+\left|\mu_{2}\right|+\cdots+\left|\mu_{n}\right|}{n}
$$

and

$$
\left|\mu_{1} \mu_{2} \cdots \mu_{n}\right|^{\frac{1}{n}}
$$

Since arithmetic mean is greater than or equal to geometric mean it follows that

$$
\begin{gathered}
\frac{\left|\mu_{1}\right|+\left|\mu_{2}\right|+\cdots+\left|\mu_{n}\right|}{n} \geq\left|\mu_{1} \mu_{2} \cdots \mu_{n}\right|^{\frac{1}{n}} . \\
\frac{\left|\mu_{1}\right|+\left|\mu_{2}\right|+\cdots+\left|\mu_{n}\right|}{\sqrt{n}} \geq \frac{\left|\mu_{1}\right|+\left|\mu_{2}\right|+\cdots+\left|\mu_{n}\right|}{n} \geq\left|\mu_{1} \mu_{2} \cdots \mu_{n}\right|^{\frac{1}{n}} .
\end{gathered}
$$

Therefore

$$
\frac{\left|\mu_{1}\right|+\left|\mu_{2}\right|+\cdots+\left|\mu_{n}\right|}{\sqrt{n}} \geq\left|\mu_{1} \mu_{2} \cdots \mu_{n}\right|^{\frac{1}{n}}
$$

implies

$$
\begin{aligned}
& \frac{n\left|\mu_{1}\right|}{\sqrt{n}} \geq|\operatorname{det}(A)|^{\frac{1}{n}} . \\
& \left|\mu_{1}\right| \geq \frac{|\operatorname{det}(A)|^{\frac{1}{n}}}{\sqrt{n}} .
\end{aligned}
$$

\subsection{Bounds for distance energy of graph.}

Lemma 2.4. If $G$ is a graph with $n$ vertices and $m$ edges and $A$ is the adjacency distance matrix which is non-singular then

$$
n|\operatorname{det}(A)|^{\frac{1}{n}} \leq \mathcal{E}_{D}(G) \leq \frac{2 M n}{|\operatorname{det}(A)|^{\frac{1}{n}}} .
$$

Proof. Using inequality of arithmetic and geometric mean of $\left|\mu_{1}\right|,\left|\mu_{2}\right|, \cdots,\left|\mu_{n}\right|$ we have

So,

$$
\frac{\left|\mu_{1}\right|+\left|\mu_{2}\right|+\cdots+\left|\mu_{n}\right|}{n} \geq\left|\mu_{1} \mu_{2} \cdots \mu_{n}\right|^{\frac{1}{n}} .
$$

$$
\mathcal{E}_{D}(G) \geq n|\operatorname{det}(A)|^{\frac{1}{n}}
$$

From $\frac{\left|\mu_{1}\right|+\left|\mu_{2}\right|+\cdots+\left|\mu_{n}\right|}{n} \geq|\operatorname{det}(A)|^{\frac{1}{n}}$ gives $\left|\mu_{1}\right| \geq|\operatorname{det}(A)|^{\frac{1}{n}}$. So,

$$
\left|\mu_{1}\right| \sum_{i=1}^{n}\left|\mu_{i}\right| \geq|\operatorname{det}(A)|^{\frac{1}{n}} \sum_{i=1}^{n}\left|\mu_{i}\right| .
$$


Since $\left|\mu_{i}\right| \leq\left|\mu_{1}\right| \forall i$, therefore $n\left|\mu_{1}\right|^{2} \geq|\operatorname{det}(A)|^{\frac{1}{n}} \mathcal{E}(G)$. But $\left|\mu_{1}\right|^{2} \leq 2 M$ from which we have $\mathcal{E}_{D}(G) \leq \frac{2 M n}{|\operatorname{det}(A)|^{\frac{1}{n}}}$. Thus $n|\operatorname{det}(A)|^{\frac{1}{n}} \leq \mathcal{E}_{D}(G) \leq \frac{2 M n}{|\operatorname{det}(A)|^{\frac{1}{n}}}$.

We use Holder's inequality inequality to get bounds for energy of graphs

Holder's inequality: If $x_{i j}(i=1,2, \ldots, n$ and $j=1,2,3, \ldots, n)$ is a nonnegative real numbers then $\prod_{i=1}^{n}\left(\sum_{j=1}^{n} x_{i j}\right)^{\frac{1}{n}} \geq \sum_{j=1}^{n}\left(\prod_{i=1}^{n} x_{i j}^{\frac{1}{n}}\right)$

$$
\begin{gathered}
\text { i.e., }\left(x_{11}+x_{12}+\ldots+x_{1 n}\right)^{\frac{1}{n}}\left(x_{21}+x_{22}+\ldots+x_{2 n}\right)^{\frac{1}{n}} \ldots\left(x_{n 1}+x_{n 2}+\ldots+x_{n n}\right)^{\frac{1}{n}} \geq \\
\left(x_{11}^{\frac{1}{n}} x_{21}^{\frac{1}{n}} \ldots x_{n 1}^{\frac{1}{n}}\right)+\left(x_{12}^{\frac{1}{n}} x_{22}^{\frac{1}{n}} \ldots x_{n 2}^{\frac{1}{n}}\right)+\ldots+\left(x_{1 n}^{\frac{1}{n}} x_{2 n}^{\frac{1}{n}} \ldots x_{n n}^{\frac{1}{n}}\right) .
\end{gathered}
$$

Theorem 2.5. Let $G$ be a graph with $n$ vertices and $m$ edges with $2 M \geq n$. If $A$ is a adjacency distance matrix which is non-singular then

$$
n^{\frac{n-1}{n}}|\operatorname{det}(A)|^{\frac{1}{n}} \leq \mathcal{E}_{D}(G)<\frac{(4 M)^{n^{2}}}{|\operatorname{det}(A)|^{(n-1)}} .
$$

Proof. Apply Holder's inequality using

$$
\left(\begin{array}{cccc}
x_{11} & x_{12} & \cdots & x_{1 n} \\
x_{21} & x_{22} & \cdots & x_{2 n} \\
\vdots & \vdots & \cdots & \vdots \\
x_{n 1} & x_{n 2} & \cdots & x_{n n}
\end{array}\right)=\left(\begin{array}{cccc}
1 & \frac{1}{\left|\mu_{1}\right|} & \cdots & \frac{1}{\left|\mu_{1}\right|} \\
\frac{1}{\left|\mu_{2}\right|} & 1 & \cdots & \frac{1}{\left|\mu_{2}\right|} \\
\vdots & \vdots & \cdots & \vdots \\
\frac{1}{\left|\mu_{n}\right|} & \frac{1}{\left|\mu_{n}\right|} & \cdots & 1
\end{array}\right)
$$

and simplify left hand side and right hand side of inequality separately.

$L H S=\left(1+\frac{n-1}{\left|\mu_{1}\right|}\right)^{\frac{1}{n}}\left(1+\frac{n-1}{\left|\mu_{2}\right|}\right)^{\frac{1}{n}} \ldots\left(1+\frac{n-1}{\left|\mu_{n}\right|}\right)^{\frac{1}{n}} \leq\left(1+\frac{n-1}{\left|\mu_{1}\right|}\right)\left(1+\frac{n-1}{\left|\mu_{2}\right|}\right) \ldots\left(1+\frac{n-1}{\left|\mu_{n}\right|}\right)$.

Since $2 M \geq n>(n-1)$ it follows that

. But

$$
L H S<\left(1+\frac{2 M}{\left|\mu_{1}\right|}\right)\left(1+\frac{2 M}{\left|\mu_{2}\right|}\right) \ldots\left(1+\frac{2 M}{\left|\mu_{n}\right|}\right)
$$

So

$$
\left|\mu_{i}\right| \leq \sqrt{2 M} \leq 2 M \Rightarrow 1 \leq \frac{2 M}{\left|\mu_{i}\right|} \forall i
$$

$$
\begin{aligned}
L H S & <\left(\frac{2 M}{\left|\mu_{1}\right|}+\frac{2 M}{\left|\mu_{1}\right|}\right)\left(\frac{2 M}{\left|\mu_{2}\right|}+\frac{2 M}{\left|\mu_{2}\right|}\right) \ldots\left(\frac{2 M}{\left|\mu_{n}\right|}+\frac{2 M}{\left|\mu_{n}\right|}\right) \\
& =\left(\frac{4 M}{\left|\mu_{1}\right|}\right)\left(\frac{4 M}{\left|\mu_{2}\right|}\right) \ldots\left(\frac{4 M}{\left|\mu_{n}\right|}\right) \\
& =\frac{(4 M)^{n}}{\left|\mu_{1} \mu_{2} \ldots \mu_{n}\right|}=\frac{(4 M)^{n}}{|\operatorname{det}(A)|}
\end{aligned}
$$




$$
\begin{aligned}
R H S & =\frac{1}{\left|\mu_{2}\right|^{\frac{1}{n}}\left|\mu_{3}\right|^{\frac{1}{n}} \ldots\left|\mu_{n}\right|^{\frac{1}{n}}}+\frac{1}{\left|\mu_{1}\right|^{\frac{1}{n}}\left|\mu_{3}\right|^{\frac{1}{n}} \ldots\left|\mu_{n}\right|^{\frac{1}{n}}}+\ldots+\frac{1}{\left|\mu_{1}\right|^{\frac{1}{n}}\left|\mu_{2}\right|^{\frac{1}{n}} \ldots\left|\mu_{n-1}\right|^{\frac{1}{n}}} \\
& =\frac{\left|\mu_{1}\right|^{\frac{1}{n}}}{\left|\mu_{1} \mu_{2} \ldots \mu_{n}\right|^{\frac{1}{n}}}+\frac{\left|\mu_{2}\right|^{\frac{1}{n}}}{\left|\mu_{1} \mu_{2} \ldots \mu_{n}\right|^{\frac{1}{n}}}+\ldots+\frac{\left|\mu_{n}\right|^{\frac{1}{n}}}{\left|\mu_{1} \mu_{2} \ldots \mu_{n}\right|^{\frac{1}{n}}} \\
& =\frac{1}{|\operatorname{det}(A)|^{\frac{1}{n}}} \sum_{i=1}^{n}\left|\mu_{i}\right|^{\frac{1}{n}}
\end{aligned}
$$

Therefore

$$
\frac{1}{|\operatorname{det}(A)|^{\frac{1}{n}}} \sum_{i=1}^{n}\left|\mu_{i}\right|^{\frac{1}{n}}<\frac{(4 M)^{n}}{|\operatorname{det}(A)|}
$$

and

$$
\sum_{i=1}^{n}\left|\mu_{i}\right|^{\frac{1}{n}}<\frac{(4 M)^{n}}{|\operatorname{det}(A)|^{\left(1-\frac{1}{n}\right)}}
$$

But

$$
\left(\sum_{i=1}^{n}\left|\mu_{i}\right|\right)^{\frac{1}{n}} \leq \sum_{i=1}^{n}\left|\mu_{i}\right|^{\frac{1}{n}}
$$

Hence

$$
\left(\sum_{i=1}^{n}\left|\mu_{i}\right|\right)^{\frac{1}{n}}<\frac{(4 M)^{n}}{|\operatorname{det}(A)|^{\left(\frac{n-1}{n}\right)}}
$$

and

$$
\mathcal{E}_{D}(G)<\frac{(4 M)^{n^{2}}}{|\operatorname{det}(A)|^{(n-1)}} .
$$

To get lower bound we apply Holder's inequality using the substitution

$$
\begin{gathered}
\left(\begin{array}{cccc}
x_{11} & x_{12} & \cdots & x_{1 n} \\
x_{21} & x_{22} & \cdots & x_{2 n} \\
\vdots & \vdots & \cdots & \vdots \\
x_{n 1} & x_{n 2} & \cdots & x_{n n}
\end{array}\right)=\left(\begin{array}{cccc}
\left|\mu_{1}\right| & \left|\mu_{1}\right| & \cdots & \left|\mu_{1}\right| \\
\left|\mu_{2}\right| & \left|\mu_{2}\right| & \cdots & \left|\mu_{2}\right| \\
\vdots & \vdots & \cdots & \vdots \\
\left|\mu_{n}\right| & \left|\mu_{n}\right| & \cdots & \left|\mu_{n}\right|
\end{array}\right) \\
\left(n\left|\mu_{1}\right|\right)^{\frac{1}{n}}+\left(n\left|\mu_{2}\right|\right)^{\frac{1}{n}}+\cdots+\left(n\left|\mu_{n}\right|\right)^{\frac{1}{n}} \geq n\left(\left|\mu_{1}\right|\left|\mu_{2}\right| \cdots\left|\mu_{n}\right|\right)^{\frac{1}{n}} . \\
\left|\mu_{1}\right|^{\frac{1}{n}}+\left|\mu_{2}\right|^{\frac{1}{n}}+\cdots+\left|\mu_{n}\right|^{\frac{1}{n}} \geq n^{\frac{n-1}{n}}(|\operatorname{det}(A)|)^{\frac{1}{n}}
\end{gathered}
$$

But $\left(\left|\mu_{1}\right|+\left|\mu_{2}\right|+\cdots+\left|\mu_{n}\right|\right) \geq\left|\mu_{1}\right|^{\frac{1}{n}}+\left|\mu_{2}\right|^{\frac{1}{n}}+\cdots+\left|\mu_{n}\right|^{\frac{1}{n}}$. Therefore

$$
\mathcal{E}_{D}(G) \geq n^{\frac{n-1}{n}}|\operatorname{det}(A)|^{\frac{1}{n}}
$$

Combining above bounds we have, $n^{\frac{n-1}{n}}|\operatorname{det}(A)|^{\frac{1}{n}} \leq \mathcal{E}_{D}(G)<\frac{(4 m)^{n^{2}}}{|\operatorname{det}(A)|^{(n-1)}}$. 


\subsection{Lower and upper bound for distance energy of graph.}

Theorem 2.6. Let $G$ be a graph with $n(\geq 2)$ vertices and $m$ edges with $2 M \geq n$ then

$$
\mathcal{E}_{D}(G) \geq \frac{2 M}{n}+\left(\frac{|\operatorname{det}(A)|}{\frac{2 M}{n}}\right)^{\frac{1}{n-1}} .
$$

Proof. Apply arithmetic mean and geometric mean inequality to real numbers $\left|\mu_{2}\right|,\left|\mu_{3}\right|, \cdots,\left|\mu_{n}\right|$ for $(n-1)$ terms,

$$
\begin{gathered}
\frac{\left|\mu_{2}\right|+\left|\mu_{3}\right|+\cdots+\left|\mu_{n}\right|}{n-1} \geq\left|\mu_{2} \mu_{3} \cdots \mu_{n}\right|^{\frac{1}{n-1}} \\
\left(\left|\mu_{2}\right|+\left|\mu_{3}\right|+\cdots+\left|\mu_{n}\right|\right) \geq \frac{\left|\mu_{2}\right|+\left|\mu_{3}\right|+\cdots+\left|\mu_{n}\right|}{n-1} \geq\left|\mu_{2} \mu_{3} \cdots \mu_{n}\right|^{\frac{1}{n-1}} .
\end{gathered}
$$

So,

$$
\mathcal{E}(G)-\left|\mu_{1}\right| \geq \frac{\left|\mu_{1} \mu_{2} \cdots \mu_{n}\right|^{\frac{1}{n-1}}}{\left|\mu_{1}\right| \frac{1}{n-1}}
$$

And

$$
\mathcal{E}_{D}(G) \geq\left|\mu_{1}\right|+\frac{|\operatorname{det}(A)|^{\frac{1}{n-1}}}{\left|\mu_{1}\right| \frac{1}{n-1}} .
$$

Let $\left|\mu_{1}\right|=x$ and $\Psi(x)=x+\frac{|\operatorname{det}(A)|^{\frac{1}{n-1}}}{x^{\frac{1}{n-1}}}$. We shall minimize the function by finding $\Psi^{\prime}(x)$ and $\Psi^{\prime \prime}(x)$. At maxima or minima $\Psi^{\prime}(x)=0$ which gives $1-\frac{|\operatorname{det}(A)|^{\frac{1}{n-1}}}{n-1} x^{-\frac{n}{n-1}}=0$. Thus the function $\Psi(x)$ attains maxima or minima at $x=\frac{|\operatorname{det}(A)|^{\frac{1}{n}}}{(n-1)^{\frac{n-1}{n}}}$. At this point, $\Psi^{\prime \prime}(x)=\frac{n}{(n-1)^{2}}|\operatorname{det}(A)|^{\frac{1}{n-1}} x^{\frac{1-2 n}{n-1}} \geq 0$. This means the function attains the minimum value at this point. The minimum value is

$$
\Psi\left(\frac{|\operatorname{det}(A)|^{\frac{1}{n}}}{(n-1)^{\frac{n-1}{n}}}\right)=\frac{n|\operatorname{det}(A)|^{\frac{1}{n}}}{(n-1)^{\frac{(n-1)}{n}}} .
$$

But the function is increasing in the interval $\frac{|\operatorname{det}(A)|^{\frac{1}{n}}}{(n-1)^{\frac{n-1}{n}}} \leq|\operatorname{det} A|^{\frac{1}{n}} \leq \frac{2 M}{n} \leq\left|\mu_{1}\right| \leq$ $\sqrt{2 M}$.

$$
\begin{gathered}
\mathcal{E}_{D}(G) \geq \Psi\left(\frac{2 M}{n}\right) . \\
\mathcal{E}_{D}(G) \geq \frac{2 M}{n}+\left(\frac{|\operatorname{det}(A)|}{\frac{2 M}{n}}\right)^{\frac{1}{n-1}} .
\end{gathered}
$$


Theorem 2.7. Let $G$ be a graph with $n(\geq 3)$ vertices and $m$ edges with $2 M \geq n$ then

$$
\mathcal{E}_{D}(G) \geq \frac{2 M}{n}+\frac{(n-2)^{\frac{1}{n}}|\operatorname{det}(A)|^{\frac{n-1}{n(n-2)}}}{\left(\frac{2 M}{n}\right)^{\frac{1}{n-2}}} .
$$

Proof. Apply arithmetic mean and geometric mean inquality to real numbers $\left.\left|\mu_{2}\right|,\left|\mu_{3}\right|, \cdots, \mid \mu_{n-1}\right) \mid$ for $(n-2)$ terms,

$$
\begin{gathered}
\frac{\left|\mu_{2}\right|+\left|\mu_{3}\right|+\cdots+\left|\mu_{n-1}\right|}{n-2} \geq\left|\mu_{2} \mu_{3} \cdots \mu_{n-1}\right|^{\frac{1}{n-2}} . \\
\left(\left|\mu_{2}\right|+\left|\mu_{3}\right|+\cdots+\left|\mu_{n-1}\right|\right) \geq \frac{\left|\mu_{2}\right|+\left|\mu_{3}\right|+\cdots+\left|\mu_{n-1}\right|}{n-2} \geq\left|\mu_{2} \mu_{3} \cdots \mu_{n-1}\right|^{\frac{1}{n-2}} .
\end{gathered}
$$

So,

$$
\begin{gathered}
\mathcal{E}(G)-\left|\mu_{1}\right|-\left|\mu_{n}\right| \geq \frac{\left|\mu_{1} \mu_{2} \cdots \mu_{n}\right|^{\frac{1}{n-2}}}{\left|\mu_{1} \mu_{n}\right| \frac{1}{n-2}}, \\
\mathcal{E}_{D}(G) \geq\left|\mu_{1}\right|+\left|\mu_{n}\right|+\frac{|\operatorname{det}(A)|^{\frac{1}{n-2}}}{\left|\mu_{1} \mu_{n}\right| \frac{1}{n-2}} .
\end{gathered}
$$

Let $\left|\mu_{1}\right|=x,\left|\mu_{n}\right|=y$ and $g(x, y)=x+y+\frac{|\operatorname{det}(A)|^{\frac{1}{n-2}}}{(x y)^{\frac{1}{n-2}}}$. Using partial differentiation we minimize the function by finding $g_{x}(x, y), g_{y}(x, y), g_{x x}(x, y), g_{y y}(x, y)$, $g_{x y}(x, y)$ and $\Delta=g_{x x} g_{y y}-g_{x y}^{2}$.

$$
\begin{gathered}
g_{x}=1-\frac{|\operatorname{det}(A)|^{\frac{1}{n-2}}}{n-2}(x y)^{\frac{1-n}{n-2}} y, \\
g_{y}=1-\frac{|\operatorname{det}(A)|^{\frac{1}{n-2}}}{n-2}(x y)^{\frac{1-n}{n-2}} x, \\
g_{x x}=-\frac{y^{2}(1-n)|\operatorname{det}(A)|^{\frac{1}{n-2}}}{(n-2)^{2}}(x y)^{\frac{3-2 n}{n-2}}, \\
g_{y y}=-\frac{x^{2}(1-n)|\operatorname{det}(A)|^{\frac{1}{n-2}}}{(n-2)^{2}}(x y)^{\frac{3-2 n}{n-2}}, \\
g_{x y}=-\frac{|\operatorname{det}(A)|^{\frac{1}{n-2}}}{n-2}\left((x y)^{\frac{1-n}{n-2}}+y \frac{n-1}{n-2}(x y)^{\frac{3-2 n}{n-2}}\right), \\
\Delta=\frac{(x y)^{2}(1-n)^{2}|\operatorname{det}(A)|^{\frac{2}{n-2}}}{(n-2)^{4}}(x y)^{\frac{6-4 n}{n-2}}-\frac{|\operatorname{det}(A)|^{\frac{2}{n-2}}}{(n-2)^{2}}\left((x y)^{\frac{1-n}{n-2}}+y \frac{n-1}{n-2}(x y)^{\frac{3-2 n}{n-2}}\right)^{2} .
\end{gathered}
$$

At maxima or minima $g_{x}=0, g_{y}=0$ which gives

$$
(x y)^{\frac{1-n}{n-2}} y=\frac{n-2}{|\operatorname{det}(A)|^{\frac{1}{n-2}}}
$$


and

$$
(x y)^{\frac{1-n}{n-2}} x=\frac{n-2}{|\operatorname{det}(A)|^{\frac{1}{n-2}}} .
$$

Solving these equations gives

$$
x=y=\frac{|\operatorname{det}(A)|^{\frac{1}{n}}}{(n-2)^{\frac{n-2}{n}}} .
$$

Thus the function $g(x, y)$ attains maxima or minima at

$$
x=y=\frac{|\operatorname{det}(A)|^{\frac{1}{n}}}{(n-2)^{\frac{n-2}{n}}},
$$

At this point, $g_{x x}$ and $g_{y y}$ are greater than equal to zero. Further $\Delta \leq 0$. This means that the function attains the minimum value at this point. The minimum value is given by,

$$
g\left(\frac{|\operatorname{det}(A)|^{\frac{1}{n}}}{(n-2)^{\frac{n-2}{n}}}, \frac{|\operatorname{det}(A)|^{\frac{1}{n}}}{(n-2)^{\frac{n-2}{n}}}\right) .
$$

Since $2 M \geq n, g(x, y)$ increases in the interval

and

$$
|\operatorname{det}(A)|^{\frac{1}{n}} \leq \frac{2 M}{n} \leq x \leq \sqrt{2 M}
$$

$$
0 \leq y \leq|\operatorname{det}(A)|^{\frac{1}{n}} \leq \frac{2 M}{n} \leq \sqrt{2 M}
$$

At

$$
\begin{gathered}
y=\frac{|\operatorname{det}(A)|^{\frac{1}{n}}}{(n-2)^{\frac{n-2}{n}}}, \\
g(x, y)=x+\frac{(n-2)^{\frac{1}{n}}|\operatorname{det}(A)|^{\frac{n-1}{n(n-2)}}}{x^{\frac{1}{n-2}}} .
\end{gathered}
$$

Therefore,$$
\mathcal{E}_{D}(G) \geq g\left(x, \frac{|\operatorname{det}(A)|^{\frac{1}{n}}}{(n-2)^{\frac{n-2}{n}}}\right) \geq g\left(\frac{2 M}{n}, \frac{|\operatorname{det}(A)|^{\frac{1}{n}}}{(n-2)^{\frac{n-2}{n}}}\right) .
$$

Hence,

$$
\mathcal{E}_{D}(G) \geq \frac{2 M}{n}+\frac{(n-2)^{\frac{1}{n}}|\operatorname{det}(A)|^{\frac{n-1}{n(n-2)}}}{\left(\frac{2 M}{n}\right)^{\frac{1}{n-2}}} .
$$

Theorem 2.8. Let $G$ be a graph with $n \geq 2$ vertices, $m$ edges and $G$ is a nonsingular graph then

$$
\mathcal{E}_{D}(G) \leq \sqrt{2 M}+\frac{(n-1)(2 M)}{|\operatorname{det}(A)|^{\frac{1}{n}}}
$$


Proof. We know that $\left|\mu_{1}\right| \geq|\operatorname{det}(A)|^{\frac{1}{n}}$, which implies

$$
\left|\mu_{1}\right| \sum_{i=2}^{n}\left|\mu_{i}\right| \geq|\operatorname{det}(A)|^{\frac{1}{n}} \sum_{i=2}^{n}\left|\mu_{i}\right| .
$$

Since $\left|\mu_{i}\right| \leq\left|\mu_{1}\right| \forall i$, therefore

$$
(n-1)\left|\mu_{1}\right|^{2} \geq|\operatorname{det}(A)|^{\frac{1}{n}}\left(\mathcal{E}(G)-\left|\mu_{1}\right|\right) .
$$

Thus

$$
\mathcal{E}_{D}(G) \leq\left|\mu_{1}\right|+\frac{(n-1)\left|\mu_{1}\right|^{2}}{|\operatorname{det}(A)|^{\frac{1}{n}}}
$$

Let $\left|\mu_{1}\right|=x$ and $f(x)=x+\frac{(n-1) x^{2}}{|\operatorname{det}(A)|^{\frac{1}{n}}}$. At maxima or minima $f^{\prime}(x)=0$ which gives

$$
1+\frac{(n-1) 2 x}{|\operatorname{det}(A)|^{\frac{1}{n}}}=0
$$

Hence the function attains maximum or minimum value at

$$
x=-\frac{|\operatorname{det}(A)|^{\frac{1}{n}}}{2(n-1)} .
$$

Since $f^{\prime \prime}(x)=\frac{2(n-1)}{|\operatorname{det}(A)|^{\frac{1}{n}}}>0$ the function attains minimum value at this point.

The minimum value

$$
f\left(-\frac{|\operatorname{det}(A)|^{\frac{1}{n}}}{2(n-1)}\right)=-\frac{|\operatorname{det}(A)|^{\frac{1}{n}}}{2(n-1)}+\frac{|\operatorname{det}(A)|^{\frac{1}{n}}}{4(n-1)}=-\frac{|\operatorname{det}(A)|^{\frac{1}{n}}}{4(n-1)} .
$$

But $f(x)$ is an increasing function in the region $-\frac{|\operatorname{det}(A)| \frac{1}{n}}{2(n-1)} \leq x \leq \sqrt{2 M}$. Hence $f(x) \leq f(\sqrt{2 M})$. Therefore

$$
\mathcal{E}_{D}(G) \leq \sqrt{2 M}+\frac{(n-1)(2 M)}{|\operatorname{det}(A)|^{\frac{1}{n}}} .
$$

\section{CONCLUDING REMARKS}

In this paper, an effort has been made to obtain new bounds for distance energy of graph in a simplest way. Are these lower and upper bounds better than Koolen-Moulton and McClelland bounds (1.1 and 1.2)? It is yet to proved and is a scope for further research. 


\section{REFERENCES}

[1] S.B Bozkurt, A. D Gügör, B. Zhou, Note on distance energy of graph,MATCH Comrhon. Math. Comput. Chem, 64(2010), 129-134.

[2] D.Cvetković and I.Gutman (eds.), Applications of Graph Spectra (Mathematical Institution, Belgrade, 2009).

[3] D. Cvetković and I.Gutman (eds.), Selected Topics on Applications of Graph Spectra, (Mathematical Institute Belgrade, 2011).

[4] A.Graovac, I.Gutman and N.Trinajstić, Topological Approach to the Chemistry of Conjugated Molecules (Springer, Berlin, Vol. 4, 1977)

[5] I.Gutman, The energy of a graph. Ber. Ber. Math-Statist. Sekt. Forschungszentrum Graz 103, pp: 1-22 (1978).

6] I.Gutman, in The energy of a graph: Old and New Results,ed.by A. Betten, A. Kohnert, R. Laue, A. Wassermann. Algebraic Combinatorics and Applications (Springer, Berlin, 2001), pp: $196-211$.

[7] I.Gutman and O.E. Polansky, Mathematical Concepts in Organic Chemistry (Springer, Berlin, 1986)

[8] H. S. Ramane, D. S Revankar, I. Gutman, S.B Rao, B. D Acharya and H. B Walikar, Bounds for distance energy of graph, Kragujevac J. Math, 31(2008) 59-68.

[9] Huiqing Liu, Mei Lu and Feng Tian, Some upper bounds for the energy of graphs Journal of Mathematical Chemistry, Vol. 41, No.1, (2007) pp: 45-57.

[10] G. Indulal, I. Gutman, A. Vijayakumar, On the distance energy of graph, MATCH Comrhon. Math. Comput. Chem., 60(2008), 461-472.

[11] Jack H. Koolen, V.Moulton and I. Gutman, Improving the McCelland inequality for $\pi$ electron energy, Chem. Phys. Lett. Vol. 320 (2000), pp: 213-216.

[12] Jack H. Koolen and V.Moulton, Maximal energy of graphs, Adv.Appl.Math, Vol. 26 (2001), pp: $47-52$.

[13] Jack H. Koolen and V.Moulton, Maximal energy of bipartite graphs, Graphs and Combinatorics, Vol. 19 (2003), pp: 131-135.

[14] B. J. McClelland, Properties of the latent root of a matrix: The estimation of $\pi$-electron energies, J. Chem. Phys. Vol. 54 (1971) pp: 640-643.

[15] Bo Zhou and Aleksandaar Ilić, on distance Spectral radius and distance energy of graphs, MATCH Comrhon. Math. Comput. Chem., 64(2010), 261-280. 